\title{
DETECTANDO LOS DESEQUILIBRIOS DOTACIONALES COMO GENERADORES DE PROBLEMAS EN LA MOVILIDAD DEL DISTRITO METROPOLITANO DE QUITO
}

\section{DETECTING ENDOWMENT IMBALANCES AS GENERATORS OF PROBLEMS IN THE MOBILITY OF THE METROPOLITAN DISTRICT OF QUITO}

\author{
Susana Astudillo-Ortegaa, ${ }^{\mathrm{a}, \mathrm{b},}$, Antonio Vázquez ${ }^{\mathrm{a}}$ \\ a Departamento de Ingeniería Topográfica y Cartográfica, Escuela Técnica Superior de Ingenieros en Topografía, Geodesia y \\ Cartografía, Universidad Politécnica de Madrid, Campus Sur, A-3, Km 7, 28031 Madrid, España. \\ tatiana.astudillo.ortega@alumnos.upm.es; antonio.vazquez.hoehne@upm.es
}

b Observatorio de la Movilidad Metropolitana, Centro de Investigación del Transporte TRANSyT, Universidad Politécnica de Madrid, 28040 Madrid, España.

\begin{abstract}
:
In metropolitan areas, daily mobility for work reasons, study, daily procedures, leisure and free time acquires great importance. This research aims to build a methodology for the analysis of daily mobility based on the use of intelligent systems that allow research the way to find the origin of congestion problems in urban mobility generated by imbalances in the spatial distribution of services, especially those that can be avoided with an adequate focus on the management of the provisioned location. This article addresses the analysis of the educational variable from the stages of compulsory education, in order to analyze the territorial distribution of these educational services, determine if there are areas with imbalances and define whether the provision of school units is related to the demand of the school-age population. Based on the algorithms used, it was obtained as a result that of the 1,269 neighborhoods / sectors that make up the Metropolitan District of Quito, $43 \%$ could reach an educational endowment balance, $46 \%$ tend to present a greater demand (south of the District and areas peri-urban), and the remaining $11 \%$ a greater offer (hypercenter of the city). In the article, concepts such as "forced mobility" and a partial redistribution simulation procedure (in which the terms "targeted demand", "targeted supply" and "targeted balance" are coined) are presented as inputs.
\end{abstract}

Key words: mobility, GIS, intelligent systems, imbalances, space distribution, displacements

\section{Resumen:}

En las áreas metropolitanas la movilidad cotidiana por motivos laborales, de estudio, trámites cotidianos, ocio y tiempo libre adquiere una importancia crucial. En esta investigación se pretende construir una metodología de análisis de movilidad cotidiana basada en la utilización de sistemas inteligentes que permitan investigar la forma de encontrar el origen de los problemas de congestión en la movilidad urbana generados por desequilibrios de distribución espacial de los servicios, especialmente aquellos que pueden ser evitados con una adecuada focalización en la gestión de la ubicación dotacional. El presente artículo aborda el análisis de la variable educativa a partir de las etapas de enseñanza obligatoria, con la finalidad de analizar la distribución territorial de estos servicios educativos, determinar si existen áreas con desequilibrios y definir si la dotación de unidades escolares está relacionada con la demanda de la población en edad escolar. En función de los algoritmos empleados se obtuvo como resultado que de los 1269 barrios-sectores que conforman el Distrito Metropolitano de Quito, el $43 \%$ podrían alcanzar un equilibrio dotacional educativo, el $46 \%$ tienden a presentar una mayor demanda (sur del Distrito y áreas periurbanas), y el 11\% restante una mayor oferta (hipercentro de la ciudad). En el artículo se presentan como aportaciones conceptos como el de "movilidad forzada" y un procedimiento de simulación de redistribución parcial, en donde se acuñan los términos de "demanda dirigida", "oferta dirigida" y "balance dirigido".

Palabras clave: movilidad, SIG, sistemas inteligentes, desequilibrios, distribución espacial, desplazamientos

\section{Introducción}

La intensa movilidad es una característica determinante de la modernidad. La movilidad representa una variable cuantitativa que mide la cantidad de desplazamientos que las personas realizan en un determinado ámbito geográfico en un intervalo de tiempo determinado (p. ej.: número de viajes/día, pasajeros/km/día, tiempo medio de viaje, etc.) y puede ser analizada mediante patrones espacio-temporales (Lizarraga 2012).

En América Latina los diferentes problemas de movilidad están asociados con la accidentalidad vial, la congestión vehicular, la contaminación ambiental y la inseguridad, los cuales están relacionados con el progresivo incremento del uso del transporte privado y el correspondiente decremento del colectivo (Lizarraga

*Corresponding Author: Susana Astudillo-Ortega, tatiana.astudillo.ortega@alumnos.upm.es 
2012). Alcántara (2010) considera que estos problemas enfrentados por la población están vinculados con la inequidad en el acceso físico y económico al espacio urbano, a los servicios e infraestructura, a la seguridad, a la calidad ambiental, a la comodidad y a la conveniencia.

La situación social del Ecuador, sobre todo de las grandes ciudades como el Distrito Metropolitano de Quito (DMQ), es en parte común con la de otros territorios latinoamericanos donde es evidente la falta de espacios públicos, la inseguridad, la no inclusión social y el desorden territorial (Jardon and Gierhake 2020). EI DMQ tiene una estructura urbana altamente condicionada por las limitaciones de la geografía y vulnerable ante las amenazas naturales, por lo que los problemas cotidianos y las perturbaciones episódicas de la movilidad urbana dificultan el funcionamiento de la ciudad y pueden llegar a limitar su desarrollo (Demoraes 2015).

Los estudios que han analizado la movilidad cotidiana de las personas y los problemas que conlleva, lo han realizado en base a datos censales y/o encuestas de origen-destino para determinar el motivo de desplazamiento y el tiempo de empleo en el mismo (Henry and Hubert 2000; Demoraes 2015; Duriez 2019; Vallejo 2017).

En la actualidad, Ecuador no cuenta con información estadística sobre movilidad espacial entre diversas zonas de poblamiento, lo que impide conocer las relaciones que se producen entre ellas (Fernández 2017).

El objetivo principal del artículo es desarrollar una metodología de análisis de movilidad cotidiana basada en la utilización de sistemas inteligentes que permitan investigar la forma de encontrar el origen de los problemas de congestión en la movilidad urbana desarrollados por desequilibrios de distribución espacial de los servicios, especialmente los que pueden ser evitados con una adecuada focalización en la gestión de la ubicación dotacional.

Todo un conjunto de factores puede contribuir a los problemas de movilidad, como son los laborales, los sanitarios, los de ocio y esparcimiento, los deportivos, religiosos, etc. Cuando en cualquiera de ellos se genera una movilidad que no se tendría que producir si hubiese una dotación espacialmente equilibrada, entonces cabría considerarlos como "desplazamientos forzados", inducidos por el desequilibrio y, en teoría, soslayables. La cuestión es compleja, pues cuanto más especializados sean, también serán menos evitables, más necesarios: es el caso, por ejemplo, de las distintas especialidades de enseñanzas universitarias respecto a la enseñanza primaria.

El ensayo consta de seis secciones, la primera aborda el aspecto introductorio del estudio, la segunda se centra en la problemática de la movilidad cotidiana en el DMQ y el sistema de transporte en el cual se funda, la tercera realiza una descripción de la zona de estudio, la cuarta presenta la metodología establecida para el análisis de la variable de educación, la misma que puede ser replicada para el análisis de otras variables, en la quinta sección se presentan los resultados obtenidos y en el último apartado se realiza observaciones de la investigación en conjunto, a modo de conclusiones.

\section{Problemas en la movilidad cotidiana del DMQ}

Ruiz (2013) menciona que uno de los problemas más urgentes por tratar dentro de la movilidad urbana, consiste en entender la dinámica de los desplazamientos y poder gestionarlos con la finalidad de satisfacer las necesidades de la demanda.

Dentro del Plan Maestro de Movilidad del DMQ (2009) se aborda la problemática del sistema de movilidad a partir de cinco componentes: transporte, gestión de tráfico, vialidad-infraestructura, gestión participativa del sistema de movilidad y el marco regulatorio. En cuanto a la componente transporte, se indica que el total de viajes que se producen diariamente en el $\mathrm{DMQ}$ es de 1,6 millones en transporte privado y 2,9 millones en transporte público; los viajes en transporte público están disminuyendo en una proporción promedio del 1,44\% anual. Se proyecta que de mantenerse estas condiciones al año 2025 , el $59 \%$ de los viajes se realizarían en transporte privado y el $41 \%$ en transporte público.

El hipercentro de la ciudad es la zona que concentra la mayor cantidad de viajes, siendo origen y destino del $50 \%$ de los que se realizan en transporte público, donde el $64 \%$ proviene de fuera de los límites de su ámbito geográfico, y el $36 \%$ dentro de dichos límites. Esto se debe a la gran cantidad de equipamientos urbanos y de servicios que se ubican en esta área (Vallejo 2017).

En cuanto a la variable transporte, la distribución territorial de los equipamientos de educación y la falta de una regulación sobre la asignación poblacional para su uso, genera una gran demanda de viajes que ha propiciado el incremento de la flota del transporte escolar, cuyo número es similar al de la flota del transporte público.

El componente gestión de tráfico registra altos niveles de saturación de congestión vehicular dentro del DMQ, los mismos que han superado la capacidad física de las vías en varios tramos, especialmente al interior del hipercentro y en sus vías de acceso. La situación se agrava debido a la presencia de equipamientos urbanos que incrementan el número de viajes y dan lugar a que se produzcan fricciones en los flujos vehiculares, como estacionamientos, paradas inapropiadas de buses, cruce aleatorio de peatones, dispositivos de control de tráfico deficientes, entre otros. Los efectos colaterales que trae consigo este problema son: contaminación, inseguridad vial y ruido.

Dentro del componente viabilidad tenemos que la movilidad registra deficiencias con la conectividad y capacidad necesaria para la estructuración y desarrollo adecuado de los desplazamientos de las personas, esta situación incide en el descenso del nivel de servicio de la red vial principal, la cual se encuentra saturada en un $32 \%$ (MDMQ 2009). Además, el inadecuado uso del suelo que se encuentra aledaño a los principales ejes viales ha incidido en la disminución de la capacidad vial.

A lo largo de los años el componente de gestión participativa ha originado una dispersión de responsabilidad relacionada con la movilidad al interior del $D M Q$, lo que ha dificultado la toma de decisiones adecuadas en la ejecución de planes, programas y proyectos. Además, no se ha socializado con la ciudadanía dichos proyectos, especialmente los que 
tienen que ver con la ubicación de obras relacionadas con la dotación de infraestructura y equipamiento.

En relación al marco regulatorio, el principal problema es que no se han elaborado proyectos de ordenanzas concisas y concretas donde se analicen y formulen lineamientos de política general en cuanto a la movilidad, viabilidad, tránsito y transporte público y privado en el DMQ.

En forma general dentro del DMQ son pocos los estudios que se han realizado sobre la dotación y ubicación de los equipamientos e infraestructuras dentro de la ciudad, además, la falta de calidad de los servicios hace que la población necesite desplazarse a otros barrios y/o sectores para satisfacer sus necesidades.

\section{Zona de estudio}

EI DMQ se localiza en la provincia de Pichincha, ocupa una superficie de $4.235 \mathrm{~km} 2$ y alberga al $15,5 \%$ de la población nacional (Fig. 1). Se compone de 32 parroquias urbanas y 33 parroquias rurales y suburbanas (Pinto 2013).

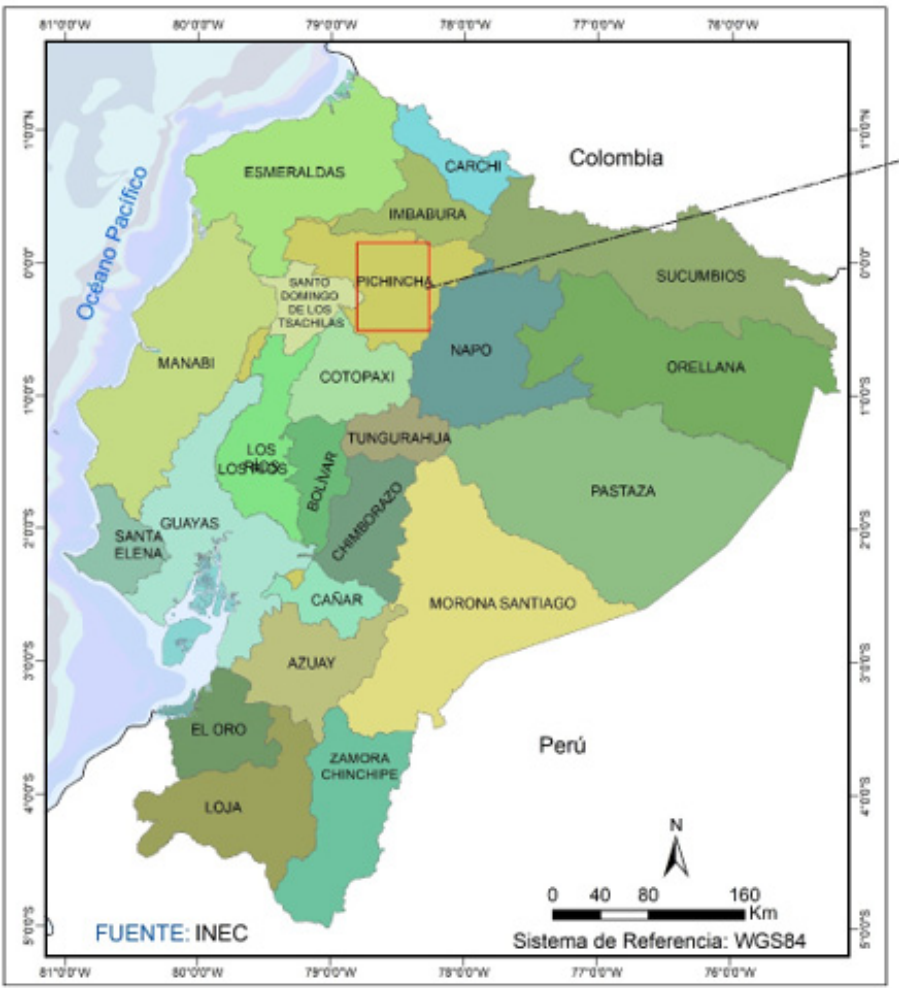

a) Provincias del Ecuador Continental

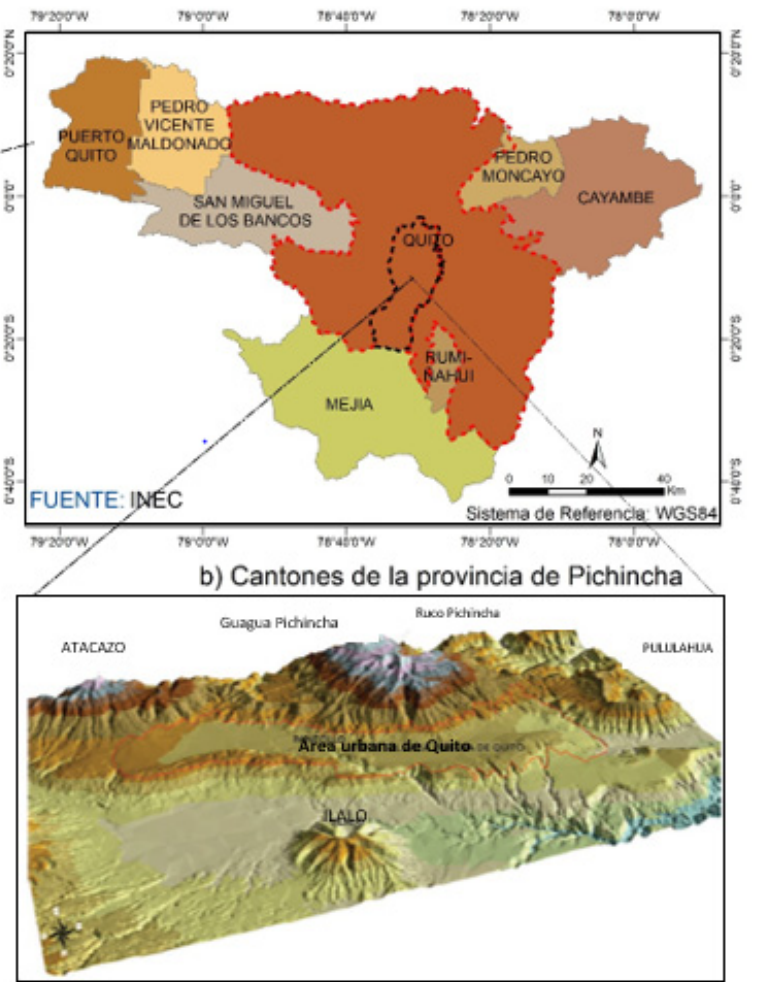

c) Región urbana de la ciudad de Quito

Figura 1: Mapa de localización del área de estudio. Fuente cartográfica: (INEC 2010).

Su estructura territorial está fuertemente condicionada por su geología y geomorfología, que han incidido históricamente en la conformación de la ciudad. La urbe quiteña se asienta sobre una grada intermedia en torno a los $2800 \mathrm{~m}$ de altitud originada por el tectonismo entre la Cordillera Occidental y la Cuenca de Tumbaco, con fallas meridianas visibles en el trayecto Quito-Tumbaco. Esta grada, colgada, presenta a la base pendientes fuertes y abruptas, con cobertura vegetal muy discontinua y un desnivel que varía entre 400 a 500 m, actualmente estas zonas se encuentran en proceso de urbanización generando que los afloramientos superficiales sean escasos. (Winckell et al. 1997).

Territorialmente la ciudad de Quito y sus áreas conurbadas forman el centro de una red urbana que estructura un conjunto diverso y heterogéneo de zonas económicas y productivas (industriales, minera, forestales y agropecuarias).

De acuerdo a los datos obtenidos el DMQ está formado por 1269 barrio-sectores, de los cuales 517 se encuentran ubicados dentro del área urbana de Quito y 752 barrios se ubican en la parte rural.
El sistema educativo dentro del área de estudio presenta un total de 1109 centros, de los cuales, el $54 \%$ son de tipo particular, $39 \%$ fiscal, $5 \%$ fiscomisional, y el $2 \%$ restante son de tipo municipal. En la Figura 2 puede observarse con claridad la clasificación de las unidades educativas a nivel urbano y rural distribuidas por su tipo de sostenimiento.

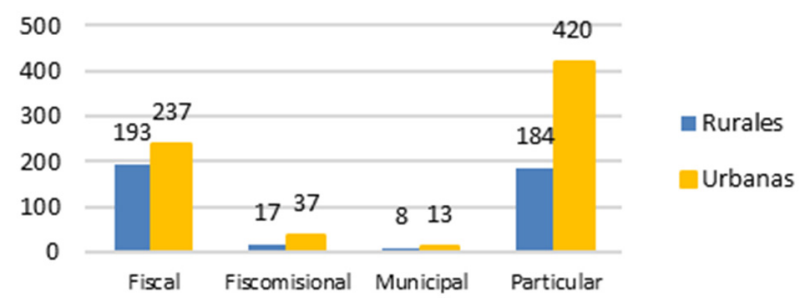

Figura 2: Unidades educativas a nivel urbano y rural clasificadas por el tipo de sostenibilidad.

\section{Material y métodos}

Para tratar la variable educación se empleó información bibliográfica, sobre todo artículos científicos y libros 
basados en movilidad urbana, calidad urbana y mecanismos de desplazamientos en el DMQ (Tabla 1). De igual forma se compilaron datos e información geográfica de diferentes instituciones gubernamentales y/u organismos para tratar la variable en análisis. En la Tabla 1 se presenta los insumos utilizados.

Tabla 1: Fuentes cartográficas utilizadas en el análisis de la variable educación.

\begin{tabular}{|c|c|c|}
\hline Material & Formato & Fuente \\
\hline $\begin{array}{c}\text { Límites administrativos } \\
\text { del DMQ }\end{array}$ & *shp & $\begin{array}{c}\text { Gobierno Abierto } \\
\text { del DMQ }\end{array}$ \\
\hline Sectores Censales & *shp & $\begin{array}{l}\text { Instituto Nacional de } \\
\text { Estadísticas y } \\
\text { Censos (INEC) }\end{array}$ \\
\hline Barrio-Sector & *shp & $\begin{array}{l}\text { Gobierno Abierto } \\
\text { del } D M Q\end{array}$ \\
\hline $\begin{array}{l}\text { Archivo Maestro de } \\
\text { Instituciones Educativas } \\
\text { Periodo } 2018-2019\end{array}$ & ${ }^{*} x \mid s x$ & $\begin{array}{l}\text { Ministerio de } \\
\text { Educación del } \\
\text { Ecuador }\end{array}$ \\
\hline $\begin{array}{c}\text { Instituciones educativas } \\
\text { del } \mathrm{DMQ}\end{array}$ & *shp & $\begin{array}{l}\text { Gobierno Abierto } \\
\text { del } D M Q\end{array}$ \\
\hline
\end{tabular}

La metodología empleada consta de dos fases, la primera corresponde al tratamiento de la información y la segunda consiste en la utilización de algoritmos que permitió el análisis espacial de los datos, su conversión y correlación de variables, mediante el uso de Sistemas de Información Geográfica (SIG).

La idea de poder realizar un balance entre la disponibilidad de plazas escolares y la necesidad de escolarización de la población cuenta con el problema inicial de que la información de las dotaciones disponibles se encuentra agrupada por los llamados barrio-sectores, mientras que la información sobre la población en edad escolar se dispone en unidades censales, normalmente de menor superficie y no siempre coincidentes.

De manera específica, en la primera fase se relacionó la distribución de la población con la distribución de los servicios de educación existentes dentro del DMQ; esta comparación espacial fue realizada en las unidades denominadas barrio-sector, para esto fue necesario una discriminación entre educación básica (5 a 12 años) y secundaria (12 a 18 años) de otro tipo de información con la finalidad de determinar si existen áreas con desequilibrio y definir si la dotación de unidades escolares está adecuadamente adaptada a la demanda de la población en edad escolar existente y, por ende, genera una serie de movimientos "forzados" que serían evitables con una óptima distribución espacial. Además, se dotó de coordenadas a las unidades educativas debido a la inexistencia de las mismas en determinadas instituciones. Con la finalidad de determinar si existen áreas con desequilibrio y definir si la dotación de unidades escolares está adecuadamente adaptada a la demanda de la población en edad escolar existente y, por ende, genera una serie de movimientos "forzados" que serían evitables con una óptima distribución espacial.

Cabe mencionar que, para dicha comparación se adaptó el censo de población en lo referente a edad educativa, para esto se partió de la estructura de unidades censales las cuales fueron convertidas a unidades por barriosector.
Las unidades censales son generalmente menores a los barrios-sectores, por lo que se incluyeron en los barriossectores cuantas unidades censales correspondían espacialmente. Para evitar duplicidades se asignó cada unidad censal a un único barrio-sector, basado en la correspondencia por mayor superficie. Así se pudo obtener el dato de la población escolarizada en cada barrio-sector y poderlo comparar con el número de estudiantes.

Como inconveniente puntual del sistema resultó que aquellos casos excepcionales de barrios-sectores muy pequeños se quedaron sin información sobre población escolarizada. Para estos casos se integraron las dotaciones al barrio-sector de mayor área. En definitiva, el problema se solventó disminuyendo parcialmente la granularidad.

Finalmente teniendo en cuenta que se trata de un problema de cantidad de tráfico extra generado, se optó como mejor indicador de desequilibrio la diferencia entre población escolarizada y dotaciones de plazas ocupadas, mas no el porcentaje.

La consideración se podría haber terminado en un simple balance entre demanda y oferta de plazas en cada unidad espacial, pero en un continuo urbano la viabilidad de trasladarse de un barrio-sector al vecino con plazas disponibles no genera problemas especiales de movilidad. Para no computar los desplazamientos de vecindad como problemas de movilidad se convino en no considerar como generadores de tráfico los casos en los que las necesidades de escolarización se pueden ver satisfechas en el barrio-sector vecino. Esto nos lleva a una situación compleja en que las disponibilidades de un barrio-sector se ven condicionados por las demandas y las ofertas más o menos satisfechas de sus vecinos: esto es, se llega a una situación de mutua dependencia.

Esto se ha resuelto mediante una aproximación de progresivos ajustes programada en Phyton donde se han considerado los siguientes pasos que simulan por reiteraciones sucesivas lo que puede ocurrir en la realidad. Cabe señalar que todo esto se realizó en la segunda fase del análisis.

1. Demanda repartida para con las unidades limítrofes: determinación de la "demanda dirigida". El primer planteamiento es que el exceso de demanda que existe en un barrio-sector se ve satisfecha con las unidades del entorno que presentan oferta. Como el proceso es reiterativo y autorregulado, en principio se ha planteado que el exceso de demanda inicial hacia cada una de las unidades colindantes es una porción simple, alícuota según el número de unidades limítrofes a la unidad con oferta. Esto se ha denominado (exceso de) "demanda dirigida" a cada una de las unidades del entorno. Así la demanda total del barrio-sector se divide entre el número de polígonos limítrofes con oferta, quedando así $n$ "demandas dirigidas", cada una con un valor total/n.

2. Oferta repartida para con las unidades limítrofes: determinación de la "oferta dirigida". Por otra parte, en los barrio-sectores con exceso de oferta, se plantea una distribución de esa oferta en función de la presión que ejerce cada una de las unidades limítrofes con exceso de demanda, expresada como la demanda dirigida calculada anteriormente. El procedimiento consiste en calcular la suma de todas 
las demandas dirigidas desde los polígonos limítrofes y respecto a esta suma determinar el valor porcentual que representa cada una y aplicarlo a la oferta total. Así para cada una de las unidades con demanda limítrofe se determinará una oferta dirigida, que a diferencia del caso anterior no será alícuota, sino proporcional.

De esta forma obtenemos para cada par de unidades limítrofes con exceso de demanda y de oferta, un valor de demanda y otro de oferta dirigidos, que desembocan en un balance "dirigido".

3. Determinación del "balance dirigido". Se calcula el balance entre la demanda dirigida y la oferta dirigida de cada par de situaciones de contacto limítrofe de oferta y demanda obteniéndose un doble "balance dirigido", como "balance dirigido de oferta" y "balance dirigido de demanda".

Este balance dirigido tendrá en el caso favorable (bien de oferta o de demanda) valor positivo y en el otro será igual a cero, originando los siguientes escenarios:

- Si la demanda es mayor: quedará un resto no satisfecho de demanda, que se imputa de vuelta como "balance dirigido de demanda" a la unidad demandante, para una posible compensación posterior. Mientras, para la ofertante, se ha consumido la oferta planteada, con lo que el valor será cero.

- Si la oferta es mayor: quedará un resto sobrante de oferta que no ha sido necesario utilizar en la compensación y que vuelve como "balance dirigido de oferta" para una posible utilización posterior. Mientras para la demandante, se ha satisfecho la demanda planteada, con lo que el valor será cero.

- Cuando sean iguales ambos valores de oferta y demanda, tendrán ambos valores cero.

4. Iteraciones hasta finalización del procedimiento. Para cada unidad de demanda se obtiene la suma de todos los "balances dirigidos de demanda" limítrofes. Igualmente, para cada unidad de oferta se obtendrá la suma de todos los "balances dirigidos de oferta" de las unidades limítrofes. Como resultado se obtendrá un mapa con nuevos valores, que pueden ser de tres tipos:

- Valores donde sigue habiendo demanda.

- Valores donde sigue habiendo oferta.

- Valores con la demanda o la oferta en cero.

Se repite todo el proceso hasta que ya no haya "ofertas dirigidas" ni "demandas dirigidas", obteniendo de esta manera el mapa definitivo de zonas con exceso de oferta, exceso de demanda y zonas con equilibrio.

\section{Resultado y discusión}

De acuerdo al análisis realizado en una primera aproximación se obtiene que dentro del DMQ 698 barrios no poseen una unidad educativa de enseñanza primaria y secundaria, lo que hace que los estudiantes tengan que desplazarse a otros barrios aledaños o alejados de su residencia para poder acceder a este servicio, lo que implica en muchos casos la utilización del transporte público o privado.

En base al indicador de desequilibrio obtenido al realizar la comparación en la unidad espacial del barrio-sector, desde la educación y desde la población resulta que 881 barrios a nivel del DMQ y 349 barrios que se encuentran en el área urbana son consideradas áreas críticas donde existe mayor demanda de la población escolarizada y no existe la suficiente oferta de unidades educativas que satisfagan las necesidades de los estudiantes. En la Figura 3.se pueden visualizar las áreas críticas a nivel del DMQ y en la Figura 4 a nivel del área urbana de Quito. Estado previo sin compensaciones de barrios limítrofes.

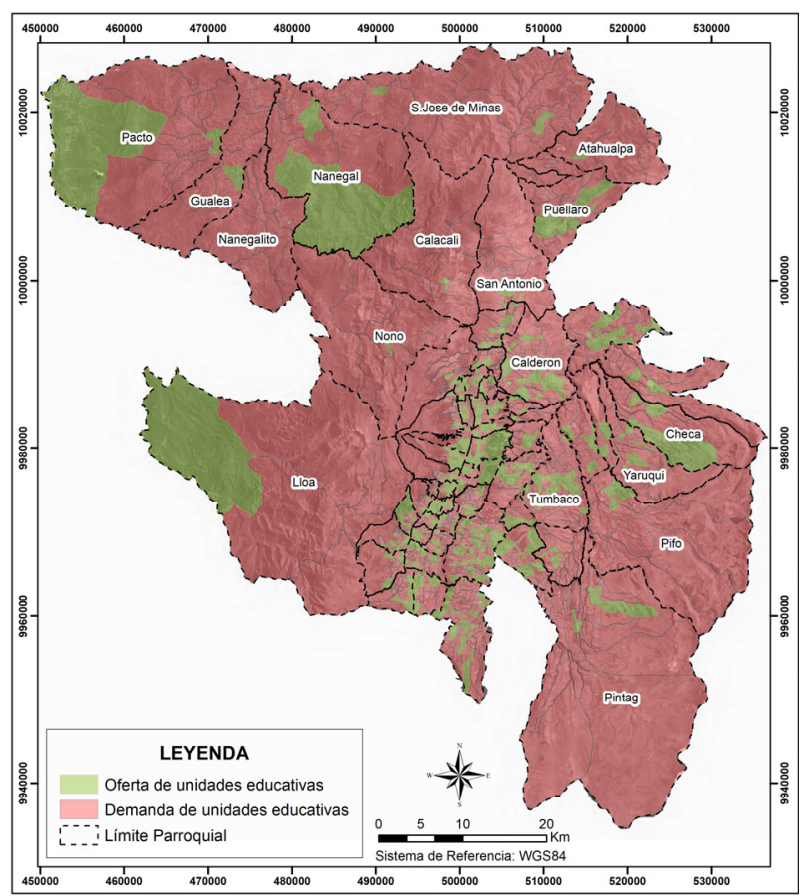

Figura 3: Mapa de los barrios que presentan oferta y demanda de unidades educativas a nivel de la población (Estado previo sin compensaciones de barrios limítrofes.

Adviértase la profusión de áreas con necesidades (en rojo) resultado de una consideración simple de oferta y demanda de plazas escolares por unidad espacial, por barrio-sector. Tal profusión atenaza, de hecho, la posibilidad de actuaciones eficaces.

Al aplicar el algoritmo elaborado (que implica la posibilidad de traslado de los estudiantes de un barriosector al vecino sin considerarlo como problema adicional de movilidad) se obtuvieron unos resultados mucho más refinados, que describen mejor el problema y que sitúan en unos 582 los barrios con demanda no satisfecha (y 215 si nos restringimos al área urbana del $D M Q$ ) según se expresa en la Tabla 2.

Con la metodología aplicada se evidencia la existencia de barrios que pueden alcanzar un equilibrio dotacional educativo y de esta manera la población vinculada a la edad escolar no tendría que desplazarse largas distancias para acceder a una institución educativa, basta con desplazarse a barrios aledaños o próximos para acceder a este servicio. 


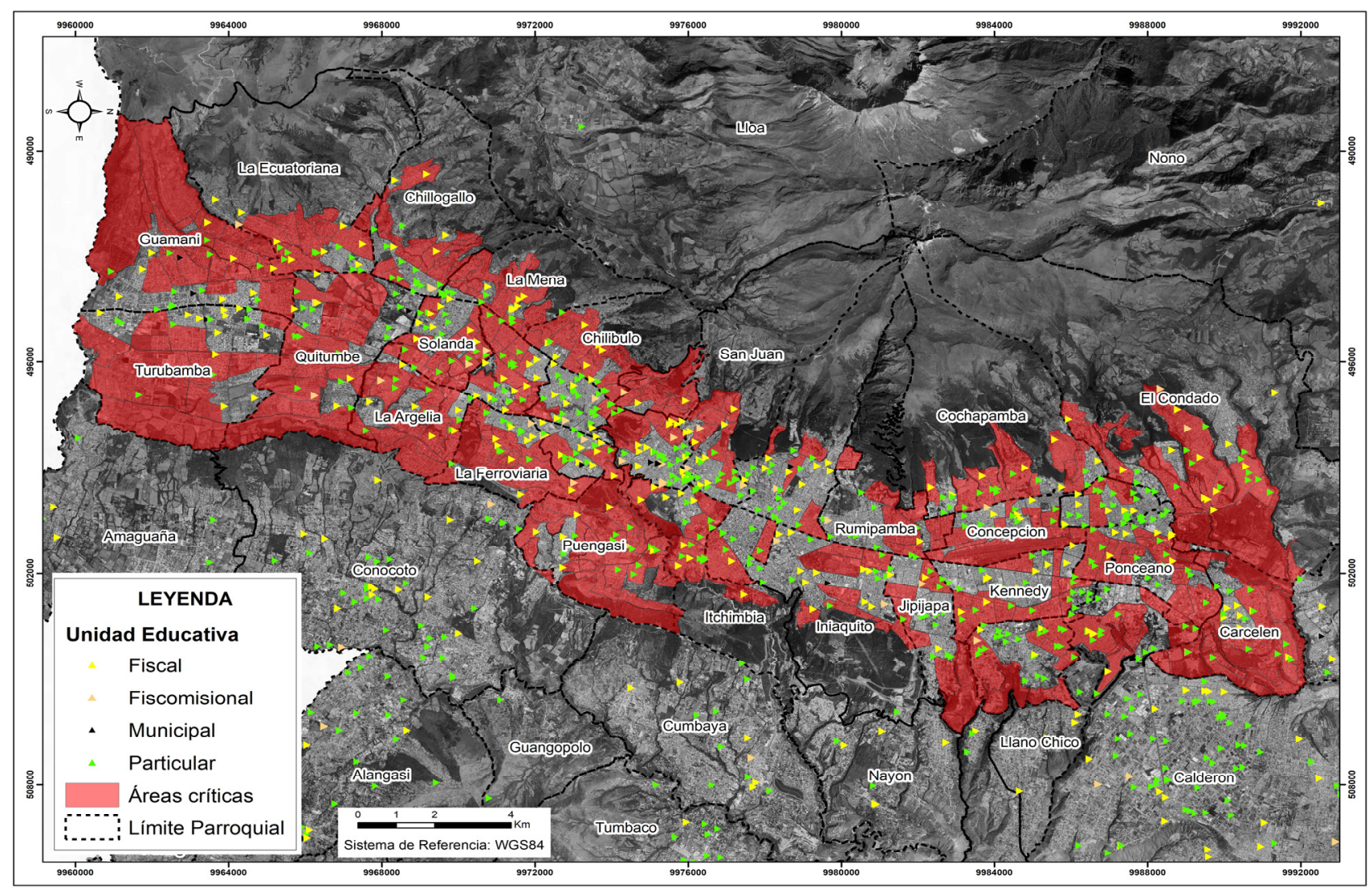

Figura 4: Mapa de los barrios que son considerados críticos a nivel del área urbana de Quito (Estado previo sin compensaciones de barrios limítrofes).

Tabla 2: Resultados obtenidos tras las iteraciones realizadas.

\begin{tabular}{c|c|c} 
Descripción & DMQ & $\begin{array}{c}\text { Área Urbana } \\
\text { del DMQ }\end{array}$ \\
\hline $\begin{array}{c}\text { Compensados } \\
\text { Barrios que siguen teniendo } \\
\text { demanda }\end{array}$ & 555 & 226 \\
Barrios que siguen teniendo oferta & 132 & 215 \\
Total de Barrios & 1269 & 518
\end{tabular}

En la Figura 5 se puede observar que a nivel del DMQ los barrios que siguen teniendo demanda de unidades educativas son aquellos que se encuentran principalmente dentro de las parroquias rurales y zonas periurbanas de la ciudad. En la Figura 6 se identifica de mejor manera que a nivel urbano del DMQ los barrios ubicados especialmente en el sur de Quito (Guamaní, La Ecuatoriana, Chillogallo, La Argelia, La Mená, Solanda, Nueva Aurora, Lucha de los Pobres, Pueblo Unido, La Ferroviaria Alta, Turubamba de Monjas, etc) y los barrios ubicados en áreas periurbanas de la ciudad (Chiribulo,Libertad, San Fernando, Alma Lojana, San Juan, Puengasí, Atucucho, Santa Rosa Singuna, Pisulí, La Josefina, Carcelén, El Condado, Comité del Pueblo, Jaime Roldós, Cochapamba Sur, entre otros) son aquellos que siguen teniendo demanda de unidades educativas, donde es necesario el planteamiento de acciones por parte del gobierno para la dotación de estos servicios debido a que así se reduciría el desplazamiento de las personas que buscan estos servicios en otros barrios, que inclusive en muchos de los casos se desplazan a polos opuestos para satisfacer sus necesidades. Finalmente, los barrios ubicados en el hipercentro de la ciudad de Quito todavía poseen una alta oferta de instituciones educativas, por lo que es necesario su regulación y control.

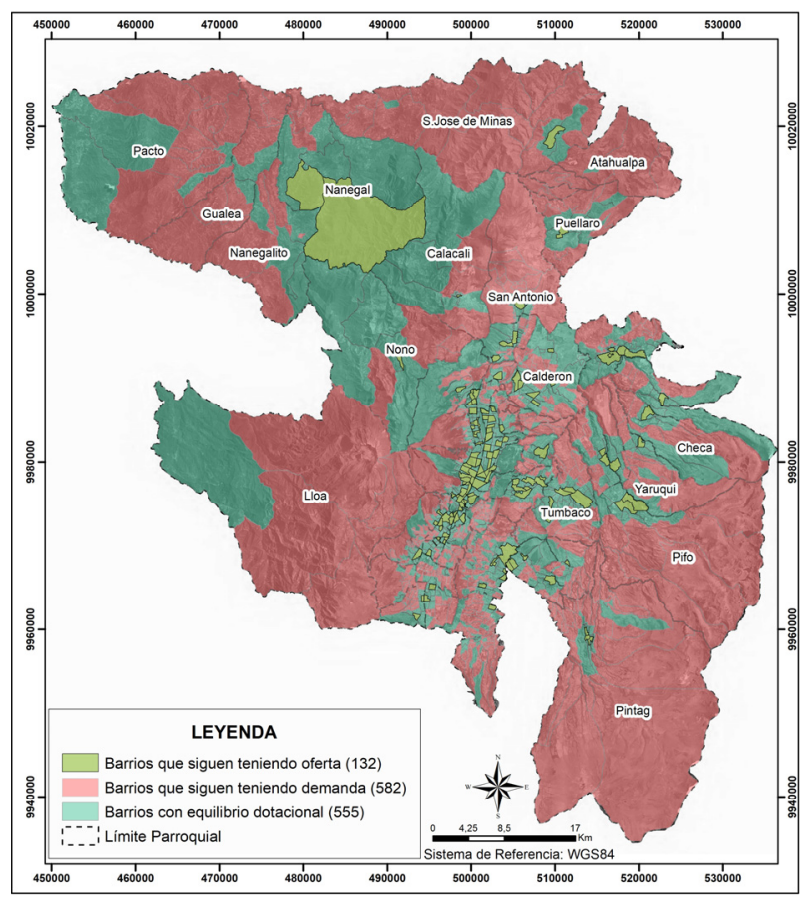

Figura 5: Mapa que contiene el resultado final, del proceso aplicado para su compensación a nivel del DMQ. 


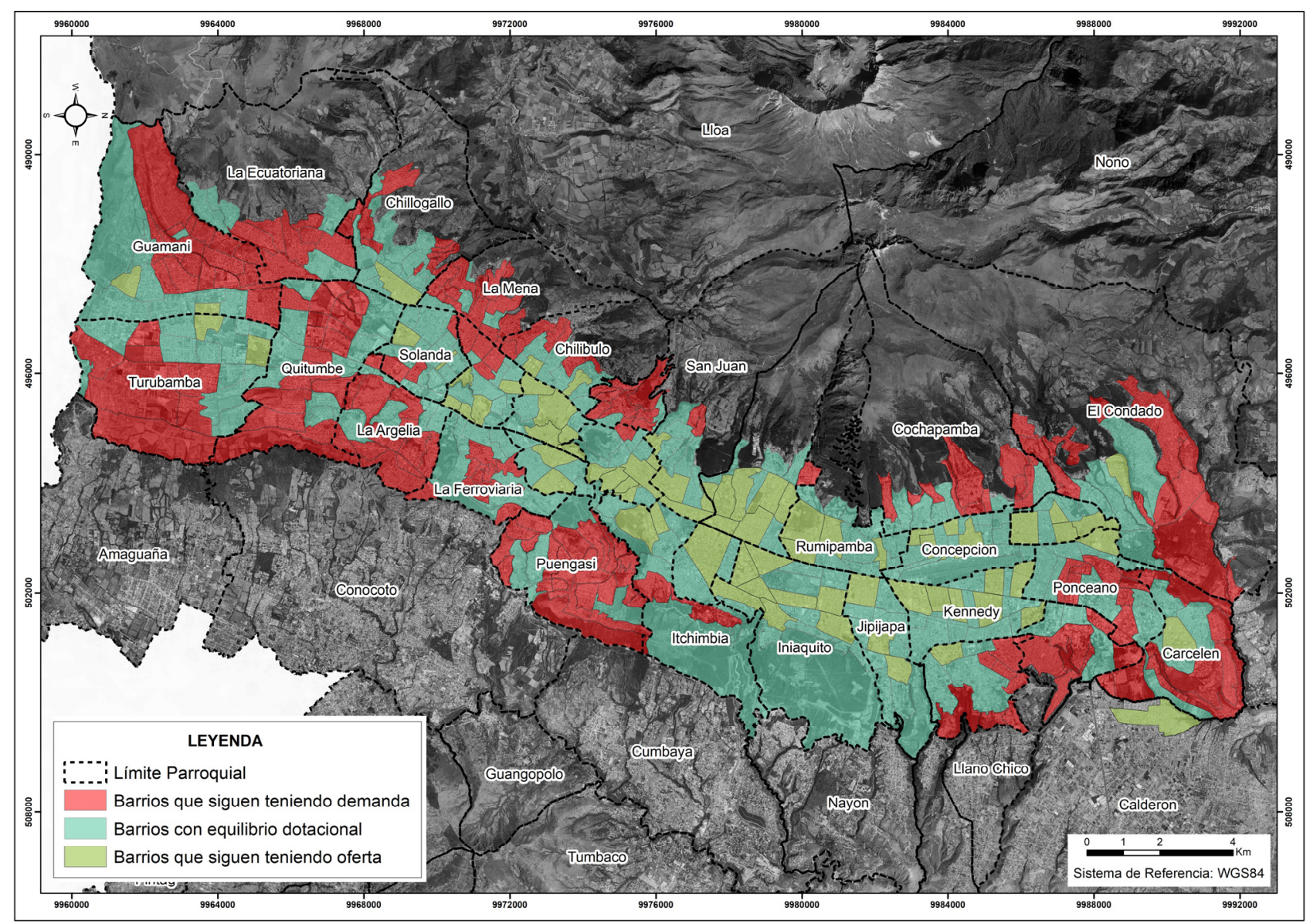

Figura 6: Mapa que contiene el resultado final del proceso aplicado para su compensación a nivel del área urbana de Quito.

\section{Conclusiones y recomendaciones}

Un problema complejo como es la relación entre unas desigualdades de demanda multifocales y de oferta multifocales se puede resolver mediante una simulación programada en diversas reiteraciones.

Los nuevos conceptos como son desplazamiento forzado, demanda dirigida, oferta dirigida balance dirigido han permitido abordar el problema de la investigación.

En función al análisis realizado de la variable educación se obtuvo como resultado que de los 1269 barriossectores que conforman el Distrito Metropolitano de Quito, el $43 \%$ podría alcanzar un cierto equilibrio dotacional educativo entre oferta y demanda, el $46 \%$ tiende a presentar una mayor demanda (sur del Distrito y áreas periurbanas), y el $11 \%$ restante una mayor oferta (hipercentro de la ciudad).

Con ello se puede concluir y localizar un desequilibrio dotacional que actúa como generador de problemas en la movilidad del DMQ, por lo que es necesario el planteamiento de acciones para reducir el impacto dentro de la ciudad.

Para verificar los resultados obtenidos se podría realizar una encuesta origen-destino a los estudiantes o a la población ubicada en los barrios-sectores donde existe mayor oferta y mayor demanda de instituciones educativas, con el fin de analizar tiempo de desplazamiento y tipo de servicio que emplea para trasladarse a la institución educativa.
Sin embargo, Ecuador no cuenta actualmente con información estadística adecuada sobre movilidad espacial, es decir, no existen censos o encuestas origendestino acerca de la movilidad entre los núcleos de población, impide conocer las relaciones que se producen entre ellos. Por ende, es necesario incorporar dentro del censo de población y vivienda, que está por desarrollarse en Ecuador, preguntas a la población como la distancia recorrida, el tiempo y dinero gastado en ellos, el medio empleado, el horario utilizado, el motivo de desplazamiento, etc., datos relevantes para este tipo de estudios.

La metodología planteada para el análisis de la variable educación puede ser replicada en otra ciudad del Ecuador o de América Latina, debido a su alta similitud y así se podrá determinar si actúa como un desequilibrio dotacional y por ende puede ser considerado como generador de problemas en la movilidad de la ciudad.

Dentro del artículo se da a conocer parte de la investigación que se está desarrollando, posteriormente se analizarán otras variables que inciden en la movilidad del $D M Q$ como generadores de problemas $y$, de igual forma, se plantearán estrategias, acciones y/o políticas de intervención y gestión a corto, mediano y a largo plazo que aporten a la seguridad y al derecho de la población a desplazarse dentro de la ciudad. 


\section{Agradecimientos}

Los autores agradecen al Observatorio de la Movilidad Metropolitana - Centro de Investigación del Transporte TRANSyT la beca otorgada para la ejecución del doctorado a partir del 01/06/2021. A las instituciones gubernamentales: Municipio del DMQ, INEC y al
Ministerio de Educación del Ecuador, por facilitar la información que se encuentra bajo su custodia. A D. Ricardo Puente por el apoyo recibido en la construcción del algoritmo en Python. Al Doctorando Francisco Cabrera por las aportaciones y comentarios realizadas al texto.

\section{References}

ALCÁNTARA, E., 2010. Análisis de la movilidad urbana. Espacio, medio ambiente y equidad. Bogotá-Colombia: Corporación Andina de Fomento (CAF).

DEMORAES, F., 2015. Movilidad, elementos esenciales y riesgos en el distrito metropolitano de Quito. Quito-Ecuador: Institut français d'études andines.

DURIEZ, T., 2019. Intra-urban forced displacement: A residential form of mobility induced by controversial constraints. Territorios, 40, pp.245-72.

FERNÁNDEZ, M., 2017. Ground public transport and accessibility, instruments for the functional analysis of the settlements system: the case of Ecuador. Estoa., 6(11), pp. 83-97.

HENRY, E. and HUBERT, J., 2000. Contrastes de la motorización y de la movilidad en las megápolis. CODATU IX (Coopération pour le Développement et l'Amélioraion des Transports Urbains et périurbains), México, DF, abril 2000.

INEC. 2010. Instituto Nacional De Estadística y Censos. Datos Censales del Distrito Metropolitano de Quito.

JARDON, C.M. and GIERHAKE, K., 2020. Social innovation and territory in municipalities: The Distrito Metropolitano de Quito case. Perfiles Latinoamericanos, 28(55), pp. 301-324.

LIZARRAGA, C., 2012. Metropolitan expansion and mobility: The case of Caracas. Eure, 38(113), pp. 99-125.

MDMQ, 2009. Plan Maestro de Movilidad para el Distrito Metropolitano de Quito 2009-2025. Quito-Ecuador.

PINTO, P., 2013. Las parroquias rurales de Quito y sus interrelaciones con el espacio urbano (Estudios sobre el Distrito Metropolitano de Quito). Revista del Instituto de la Ciudad, 2(2), pp. 71-119.

RUIZ, E., 2013. Metodología para la determinación de la movilidad en ciudades de tamaño medio: El caso de Mérida, Universidad de Extremadura - España.

VALLEJO, P., 2017. La Movilidad Urbana en Ciudades Intermedias del Ecuador. Alternativas Viables hacia la Sostenibilidad. El caso de Pujilí. Universidad de Extremadura - España.

WINCKELL, A., ZEBROWSKI, C. and SOURDAT, M., 1997. Los Paisajes Naturales del Ecuador. Quito-Ecuador. 